\title{
ORGANIZACIÓN DE LA RUTINA DIARIA EN ESTUDIANTES DE PRIMER NIVEL FORMATIVO EN LA ESCUELA DE TERAPIA OCUPACIONAL. UNIVERDIDAD DE CHILE ${ }^{1}$
}

\author{
ORGANIZATION OF THE DAILY ROUTINE IN FIRST LEVEL FORMATIVE STUDENTS IN \\ THE SCHOOL OF OCCUPATIONAL THERAPY. UNIVERDIDAD CHILE
}

\section{Silvia Gómez Lillo², Laura Rueda Castro³}

\begin{abstract}
Resumen:
Estudio no experimental y descriptivo, que analiza los patrones ocupacionales de personas específicas, en un corte transversal, desde una mirada cuanti y cualitativa. Esto significa que se examina la organización del tiempo y orquestación de los participantes y los significados que las personas atribuyen a sus ocupaciones.

Se aplica el Patrón de Idiosincrasia, propuesto por S. Cynkin, modificado por las autoras en que se observa tiempo, frecuencia, diversidad. El análisis cualitativo se realiza a través de un autoanálisis del Patrón en cuyo resultado se visualiza la descripción de las ocupaciones más importantes; el sentido de las actividades realizadas; evaluación de lo anterior de acuerdo a expectativas de vida, reformulación de propósitos y una reflexión en cuanto a cómo están viviendo sus vidas.

La importancia que se atribuye a este estudio se relaciona con la posibilidad que tienen los jóvenes de la muestra de tener una instancia de reflexión sobre su situación en el mundo y de los profesionales terapeutas ocupacionales de profundizar sus conocimientos en un tema relevante. Todo lo que está respaldado por la premisa de que el equilibrio ocupacional relativo lleva a mayor bienestar en la vida de las personas.
\end{abstract}

Palabras clave:

Patrones ocupacionales, orquestación, equilibrio ocupacional relativo.

\begin{abstract}
Experimental and descriptive study which analyses the occupational patterns of specific persons, in cross-section, from a quantitative and qualitative look. It means examining the organization of time and orchestation of the participants and the meaning that people attributed to their occupations.

Idiosyncratic Pattern is applied, proposed by S. Cynkin, modified by the authors in which is observed time, frequency and diversity.

The qualitative analysis is done through a self analysis of the pattern describing the most important occupations, the sense of performed activities, evaluation of the above according to life expectancy, reformulation of the purpose and meditation in so far as they are living their lives.

The importance attributed to this study relates to the possibility that people in the sample have an instance of reflection on their situation in the world and professional occupational therapist of deepen their knowledge in a relevant subject. All that is backed by the premise that the relative occupation balance leads to increase well-being in the live people.
\end{abstract}

Keywords:

Occupational patterns, orchestation, relative occupational balance.

Trabajo presentado en I Congreso Chileno de Terapia Ocupacional Abril 2013

2 Terapeuta Ocupacional Profesora adjunta. Coordinadora de Comité de estudios de la ocupación Escuela de Terapia Ocupacional. Facultad de Medicina Universidad de Chile. sgomez@med.uchile.cl

3 Terapeuta Ocupacional. Profesora Asociada. Integrante de Comité de estudios de la ocupación Escuela de Terapia Ocupacional. Facultad de Medicina. Universidad de Chile. Irueda@med.uchile.cl 


\section{INTRODUCCIÓN}

Destacar la vida cotidiana como un área propicia para producir conocimientos que nos acerquen a la comprensión de la estructuración de rutinas que efectúan las personas en contextos determinados, con el fin de desarrollar planes de vida individuales y colectivos, es la tarea que guía el presente estudio titulado: Organización de la rutina diaria de los estudiantes de primer año en la Escuela de Terapia Ocupacional de la Universidad de Chile.

En abstracto, la vida cotidiana pareciera ser homogénea para todos los miembros de una misma comunidad; sin embargo, cuando se la examina de cerca, es apreciable que ella tiene poco en común entre las mismas personas (Heller, 1998). Es ello lo que despierta el interés de Terapeutas Ocupacionales, en la tarea de comprender en profundidad el juego de intersubjetividades que existen en el día a día de las personas.

Se trata de una investigación cuanti-cualitativa que analiza la porcentualidad de actividades y tiempos involucrados en el quehacer de las personas participantes en este estudio; pero a la vez, este trabajo se detiene, específicamente, a examinar los significados que sus protagonistas atribuyen a las acciones que despliegan en su vida diaria.

El marco conceptual, sobre el que se elabora la propuesta, considera aspectos filosóficos, socio cognitivos, culturales y ocupacionales.

Desde una mirada filosófica, fenomenológica, la vida cotidiana describe un movimiento reflexivo, en un sentido esquemático y cinético, esto es, que simplemente regresa -constante y persistentemente- a un mismo punto de partida espacial y temporal (Giannini, 1987); que para la mayoría de los seres humanos lo constituye el domicilio y se circunscribe formando un periplo que pasa por calles, lugar de trabajo o de estudio, plazas, café, lugares de encuentros y otros lugares que se van repitiendo en el acontecer habitual. Cuando caemos en cuenta del movimiento reflexivo espaciotemporal, nos encontramos en el núcleo mismo de lo propiamente humano. (Valdés 2008).

Las ciencias, por lo general, buscan explicar hechos Ilamativos, que exceden lo habitual. Nos Ilaman la atención los eventos que son calificados como extraordinarios de acuerdo con los parámetros establecidos por cierta concepción de la realidad, ajustada a las preferencias que se derivan de dominios cognitivos predominantes en el marco de una determinada cultura (Maturana, 1994). Sin embargo, para la terapia ocupa- cional el establecer sistematizaciones del devenir cotidiano es una fuente de gran sustento teórico.

Este trabajo considera algunos argumentos que sirven de base a la posibilidad de asumir la vida cotidiana de personas y comunidades como espacio para la construcción de conocimiento social; ello amerita la concepción de las rutinas de vida como modalidad de reconstrucción de lo socioestructural (Villegas, González 2011) y, la utilización de hábitos sociales, como instancia mediante la cual se accede a lo social examinando la cotidianidad cultural de quienes constituyen la comunidad que es objeto de la atención investigativa.

Bateson, en su libro "Composing a life" (2001) define la organización de la vida, como un proceso a través del cual vamos armando mosaicos de ocupaciones que seleccionamos de acuerdo a nuestras demandas de atención y tiempo disponible. Por otra parte, la Ciencia de la Ocupación busca explicar la naturaleza de las ocupaciones y cómo ellas son orquestadas en la vida de las personas. Así, el estudio de la rutina diaria es importante para los terapeutas ocupacionales y para los cientistas de la ocupación porque:

1. Entrega un cuadro conceptual de las rutinas organizadas en el tiempo, con relación a la salud y al bienestar.

2. En la vida actual, existe la percepción de incremento del estrés e insuficiente tiempo disponible para comprometerse en ocupaciones relajantes; así las ocupaciones de tipo productivo invaden a las demás creando un desequilibrio indeseado y poco saludable (Matuska K.: 2008, 2009, 2010).

3. Los terapeutas ocupacionales, entre otros profesionales, estudian los patrones ocupacionales de las personas en un intento por identificar las características que contribuyen a su salud y bienestar.

4. Los terapeutas ocupacionales continuamente alentamos a nuestros clientes a establecer y seguir una rutina apropiada y efectiva para ellos, por lo que es necesario para este profesional estudiar más a fondo el significado de la rutina diaria a través del tiempo y cómo ésta cambia en el curso de la vida

Las personas generalmente organizan la rutina diaria en forma rítmica, cíclica y de manera de lograr la máxima armonía en el comportamiento ocupacional. En esta organización influyen diversos factores entre los 
que se cuenta el estilo psicomotor de la persona, el lugar geográfico en que habita y especialmente el medio social en que se encuentra. La organización significa priorizar sus elecciones, armonizarlas y hacerlas congruentes con sus obligaciones, intereses y en muchos casos con las personas con que convive o se relaciona. Sus elecciones cambian a través de los años principalmente influenciadas por sus responsabilidades (familia, trabajo). Ante lo anterior, no debemos olvidar que la forma en que la persona orquesta sus ocupaciones, influye en su salud y su bienestar.

Como todo lo que hacemos los seres humanos se realiza en un espacio y a través del tiempo, tampoco debemos olvidar estos conceptos, los que pueden ser medidos objetivamente, pero en el transcurso de la vida, lo que percibe la persona es el tiempo subjetivo y no el tiempo cronológico. Así, cuando alguna actividad nos entretiene, sentimos que el tiempo pasa rápidamente, al contrario de que si estamos aburridos. Por esta razón se analizará el significado de la rutina de los participantes en este estudio.

Existen variados métodos para conocer como las personas usan su tiempo (Watanabe, 1968; Cynkin, 1979; Neville, 1980, Johansson, Grupo escandinavo de Terapia Ocupacional, entre otros). En Chile tenemos una importante investigación realizado por INE-CEPAL denominada "Encuesta sobre uso del tiempo" de 1999 en que la persona relataba las actividades efectuadas el día anterior, con intervalos de 30 minutos, diferenciando actividad principal de secundaria (www.ine.cl).

Las autoras presentaron una investigación en el IV Congreso Latinoamericano de Terapia Ocupacional, realizado en Santiago de Chile en junio de 2000: Patrón de Idiosincrasia de los alumnos que ingresan a la Escuela de Terapia Ocupacional". Se eligió el método Patrón de Idiosincrasia propuesto por S. Cynkin (1990), por ser un método objetivo que disminuye en gran medida la subjetividad del participante $y$, aunque es un corte transversal, analiza las actividades realizadas en una semana normal en la vida de la persona. El tema ha sido sistematizado en publicación universitaria (2002). La investigación actual se desarrolla con el mismo método y toma en cuenta los conceptos anteriores; profundizando los aspectos cualitativos del proceso de análisis.

\section{Metodología}

Se presenta un estudio longitudinal, no experimental, descriptivo, que toma una muestra aleatoria simple de los Patrones de Idiosincrasia confeccionados por 27 estudiantes que ingresan a la carrera de Terapia Ocupacional de la Universidad de Chile en la década 2002-2012. Esta actividad se desarrolla en el marco de la asignatura Ciencia de la Ocupación y Terapia Ocupacional.

Cuantitativamente se pondera el tiempo invertido por los estudiantes en las diferentes áreas de ocupación y cualitativamente se interpretan las percepciones que ellos expresan en el análisis de su patrón de idiosincrasia.

De acuerdo a los objetivos del estudio se analiza el equilibrio relativo en la organización de la rutina diaria y la percepción que los estudiantes tienen sobre ésta.

Para la confección del Patrón de Idiosincrasia y para su posterior análisis, se utiliza la nomenclatura del Marco de trabajo 1 (AOTA 2002), que define las siguientes áreas de desempeño, hoy denominadas áreas de ocupación: AVDB, AVDI, Educación, Trabajo, Juego, Tiempo libre, y Participación Social. En el análisis de esta investigación se considera dentro del tiempo libre al juego y a la participación social.

\section{Aspectos éticos}

En el contexto de la investigación de la vida cotidiana de cualquier persona, se hace necesario saber que lo que caracteriza a la investigación cualitativa no es la perfección forzada sino la honestidad, la rigurosidad metódica, la reflexión crítica y permanente desde y sobre lo cotidiano (Villegas, González 2011). Igualmente, la contrastación de lo percibido, lo interpretado bajo la mirada de quien hizo de sujeto investigado. Es decir, no se puede llegar a hacer el análisis de los datos sin buscar que quienes nos suministraron la información la puedan aprobar previamente, por medio de un correcto proceso de consentimiento informado. Esto último, se desarrolla al inicio de la investigación. 


\section{Resultados de información cuantitativa}

El gráfico $\mathrm{N}^{\circ} 1$

Muestra el tiempo utilizado por los estudiantes en la relación de las diferentes Áreas de Desempeño

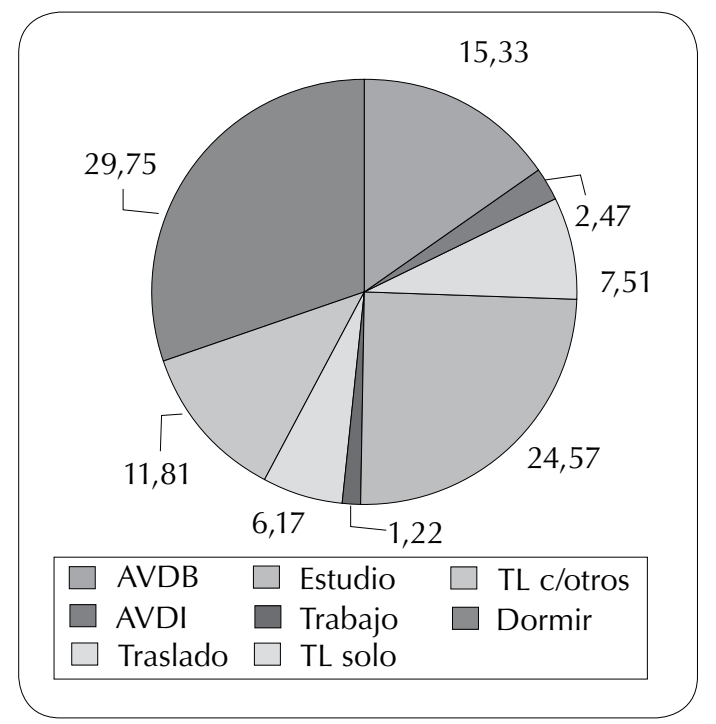

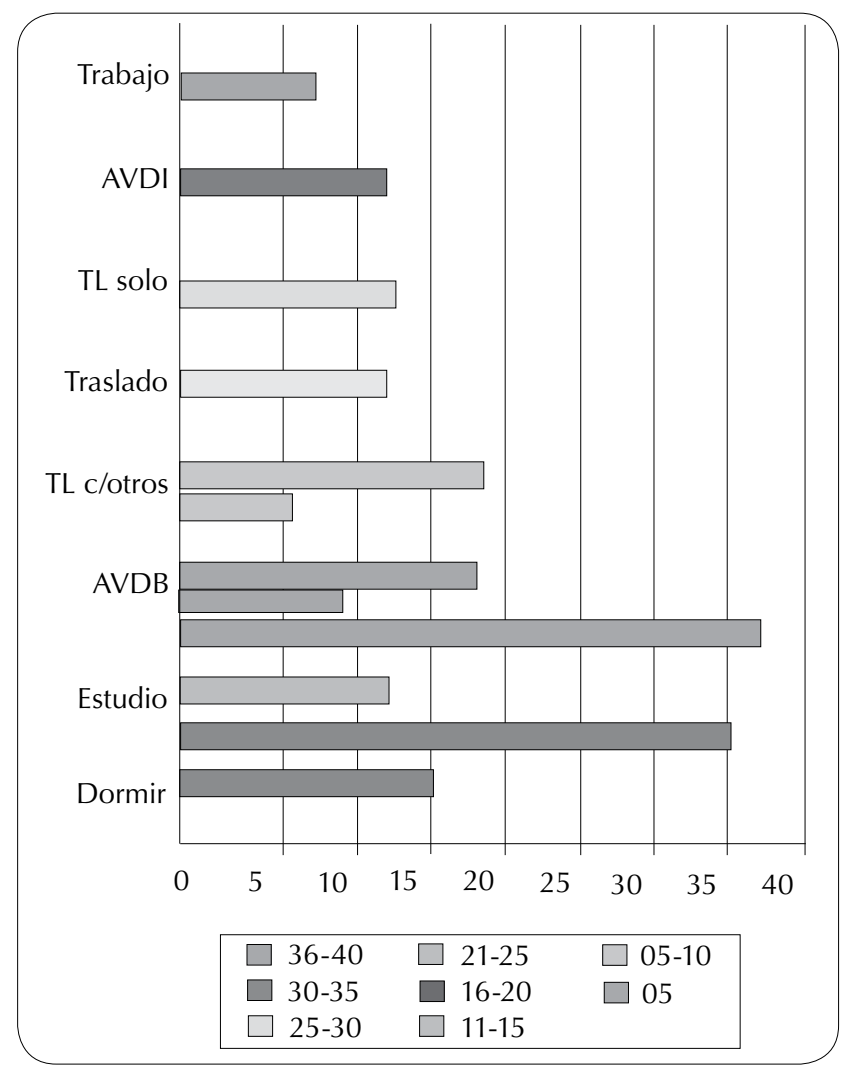

Diferencias en la utilización del tiempo de los integrantes de la muestra \% según frecuencia (lo anterior traspasado a frecuencias)

\begin{tabular}{|c|c|c|c|c|c|c|c|c|}
\hline & AVDB & AVDI & Traslado & Estudio & Trabajo & TLs & TLa & Dormir \\
\hline $0.0 \%$ & & 7 & & & 17 & & & \\
\hline $0.1-5 \%$ & & 15 & 6 & & 6 & 12 & & \\
\hline $5.1-10 \%$ & 1 & 4 & 16 & & 4 & 14 & 10 & \\
\hline $10.1-15 \%$ & 19 & 1 & 5 & 1 & & & 9 & \\
\hline $15.1-20 \%$ & 6 & & & 8 & & 1 & 8 & \\
\hline $20.1-25 \%$ & 1 & & & 13 & & & & 2 \\
\hline $25.1-30 \%$ & & & & 2 & & & 1 & 13 \\
\hline $30.1-35 \%$ & & & & 3 & & & & 9 \\
\hline$>35 \%$ & & & & 2 & & & & 3 \\
\hline$\%$ promedio & 14.5 & 3.0 & 7.5 & 24.48 & 1.9 & 6.48 & 12.96 & 28.42 \\
\hline$\%$ menor & 9.24 & 0.0 & 1.0 & 12.2 & 0.0 & 0.7 & 6.5 & 26.2 \\
\hline$\%$ mayor & 19.0 & 12.0 & 15.0 & 38.0 & 8.0 & 9.5 & 26.0 & 36.3 \\
\hline
\end{tabular}


Debido a que en el análisis cualitativo realizado por los integrantes, valoran mucho su tiempo libre, tanto en tiempo como en el contenido de éste, se presenta la siguiente tabla:

\begin{tabular}{|c|c|c|c|}
\hline \multicolumn{4}{|c|}{ NÚMERO Y FRECUENCIA DE LAS Actividades de Tiempo Libre } \\
\hline Actividad & $\mathrm{N}^{0}$ respuestas & $\mathrm{N}^{\circ}$ horas totales & Promedio de horas \\
\hline Ver televisión & 22 & 175 & 8 \\
\hline Compartir con amigos & 14 & 105 & 7,5 \\
\hline Internet/computador & 13 & 68 & 5,1 \\
\hline Deportes & 11 & 33 & 3 \\
\hline Conversar & 12 & 128 & 10 \\
\hline Escuchar música & 11 & 19,50 & 1,75 \\
\hline Compartir con familia & 9 & 61 & 6.5 \\
\hline leer & 9 & 17 & 2,15 \\
\hline chatear & 7 & 25.4 & 8,10 \\
\hline Cine & 6 & 13,45 & 2,30 \\
\hline Iglesia & 6 & 14,5 & 2,30 \\
\hline Teléfono & 6 & 6,40 & 1,30 \\
\hline Gimnasia & 5 & 19 & 3,7 \\
\hline Pololear & 5 & 36,30 & 6,10 \\
\hline Ocio & 5 & 10,30 & 2,10 \\
\hline Ver videos & 5 & 11 & 2,15 \\
\hline Oración & 3 & 10 & 3,20 \\
\hline Tocar instrumento & 3 & 3 & 1 \\
\hline Bailar & 3 & 10,20 & 3,5 \\
\hline Escuchar radio & 2 & 10,20 & 2,10 \\
\hline Participar en coro & 2 & 6 & 3 \\
\hline Manualidades & 2 & 8 & 4 \\
\hline Caminar & 1 & 1 & 1 \\
\hline Ir a mall & 1 & 2 & 2 \\
\hline
\end{tabular}

\section{Análisis de Información Cualitativa:}

La investigación cualitativa que, para Martins y Bicudo (1989), representa una comprensión particular, con foco centralizado en lo específico, lo individual y en la busca de la comprensión de los fenómenos estudiados, se ve necesaria en el presente estudio.
Se trata de producir un conocimiento basado en percepciones y subjetividades, descritas como observaciones de los participantes. Su aplicación queda justificada con la utilización de los métodos y técnicas que en estos abordajes se han generado /Romero - Salazar. 1990). En el caso de esta investigación, se produjo una dinámica cualitativa, dirigida, en primer lugar, a aportar datos de las observaciones en las nociones y creencias, 
actitudes y comportamientos en torno a la información entregada y a sus resultados.

Esta investigación trabaja con el universo de significados, aspiraciones y actitudes que corresponden a un espacio más profundo de las relaciones, de los procesos y fenómenos, que no pueden ser reducidos a la operacionalización de variables (Gonçalves, 1998).

Para operacionalizar los contenidos de lo informado se recurre a la preponderancia conceptual que emerge del análisis de los textos escritos, señalados con precisión en las observaciones y comentarios, así, se determinan 5 ejes de análisis:

1. Descripciones a partir de los datos cuantitativos

2. Retrospección de la información obtenida

3. Evaluación del proceso "Confección de Patrón de Idiosincrasia"

4. Formulación de nuevos propósitos

5. Reflexibilidad más amplia

\section{Descripciones a partir de los datos cuantitativos}

A partir de los datos numéricos que se concluyen en el análisis del tiempo y frecuencia de las actividades desarrolladas, es posible hacer una reflexión acerca del estilo de vida que de allí se desprende. Esta posibilidad de análisis cualitativo permite examinar los datos de manera objetiva, o más específicamente en forma práctica, para definir las condiciones de calidad de vida que cada cual autopercibe:

Por ejemplo:

"Las hrs. de sueño y las de productividad están dentro de los límites normales, sin haber en ningún caso sobrecarga o déficit".

"Las AVDB e I y el estudio se encuentran en equilibrio parcial con 34,63,21,83, y 20,03\% del total de la torta porcentual, como así también lo está el TL solo y el TL acompañado con 6,77 y 9,03\% del total."

"Dedico un 14\% a actividades de TL y sólo el 3\% a Participación social. AVDB 13\% que equivale a 21 hrs. semanales. AVDI 11\%".

\section{Retrospección de la información obtenida}

Mirada o examen que considera su desarrollo anterior. Apropiación y explicitación de la información obtenida para mostrar un corte transversal en la trayectoria de vida. La información obtenida sirve para encontrar sentido a las actividades y el desarrollo personal. Es la retrospección de la conciencia hacia cada quehacer diario.

Por ejemplo:

"Es satisfactorio personalmente atribuir con "argumentos" que soy más sociable de lo que pensaba y que disfruto de esto."

"El grado de compromiso en el estudio me hace invertir gran cantidad de tiempo y energía en esta ocupación aunque también en gran parte influye un factor externo por parte de la $U$ que es la obligación de mantener un rendimiento mínimo durante el año académico".

"El grado de equilibrio es un poco disparejo ya que dedico mucho tiempo a $T L$, pero lo considero necesario ya que comparto mucho con mi familia y a que realizo muchas otras actividades."

\section{Evaluación del proceso “Confección de Patrón de Idiosincrasia"}

Algunos comentarios van en dirección de evaluar en forma global, el proceso de confección del Patrón de Idiosincrasia. Se considera aquí, los componentes de la calidad de este tipo de análisis y se valora el cumplimiento con los requisitos que debe reunir una evaluación. Además, responde a las expectativas, de acuerdo a lo que ella (o)s estimen sean lo más pertinente en cada uno de los proyectos de vida.

Por ejemplo:

"En conclusión este trabajo me sirvió para ver objetivamente la composición de mi tiempo, que en sí casi todo es ocupación".

"Permite tomar conciencia acerca de los excesos y falta de ciertas actividades que si son reguladas y corregidas nos pueden beneficiar en muchos aspectos".

"Ha sido una buena herramienta para poder observar de manera gráfica la distribución de mis actividades durante la semana. Además el análisis cuantitativo de los datos obtenidos ha funcionado como un buen complemento para diagnosticar el equilibrio o desequilibrio existente en mi rutina semanal". 


\section{Formulación de nuevos propósitos}

El análisis cualitativo nos lleva también a percibir una reformulación de propósitos de vida, de algunos estudiantes. En algunos casos, como consecuencia de la retrospección anteriormente señalada. Se describen expresiones con la intención de subordinar unas necesidades a otras y adaptarse a nuevos contextos. Tienen como fin enfrentar el desequilibrio y formular un reequilibrio de las rutinas diarias. Para un nuevo esquema, consideran los propósitos como motivadores y de las actividades.

Por ejemplo:

"Por lo tanto trataré de realizar una actividad paralela mientras me traslado."

"Me gustaría desarrollar en mi TL alguna actividad física, como un deporte o caminar".

"Las modificaciones en relación a mi rutina están abocadas a reducir el tiempo de traslado desde el hogar a la $U$ o a cualquier otro lugar considerando alternativas como cambiar la forma o en casos extremos en lo que se amerite cambiando de domicilio que para mí es la mejor alternativa porque evitaría atrasos, cansancio y estrés".

\section{Reflexibilidad más amplia}

Hay formas de reflexibilidad, por parte de los participantes, producidos a partir del análisis el trabajo. Entendemos esta capacidad como un enfrentamiento que se pliega con facilidad la apropiación y explicitación de la información obtenida, a los contextos de voluntad o a la actitud de otros, en particular del tiempo y estilo de vida más globalizado del ser humano actual. Se trata de una reflexión de propósitos, ajustada a las demandas del momento histórico.

Tras analizar los contenidos producidos, hemos considerado tres reflexibilidades sucesivas, siguiendo la propuesta de Baudouin (2012): una reflexibilidad de emancipación, una reflexibilidad de búsqueda de la coherencia y una reflexibilidad sobre el poder de actuación del sujeto. Elementos de vital importancia por el impacto que genera en el desarrollo social.

Por ejemplo:

"Para mí es una bendición poder optar a una educación superior, más aún cuando se trata de una carrera que siempre quise seguir y en una casa de estudios donde me esforcé por ingresar."

"Es complejo determinar una estabilidad que permanezca en el tiempo debido a las fluctuaciones que dependen principalmente de los factores extrínsecos que pueden facilitar el DO o coartarlo de cierto modo, cabe hacer notar la tríada persona-ambiente-ocupación que aclara este concepto de cierta dependencia de la persona al entorno circundante."

"Creo que la clave para mejorar la satisfacción es sentirse a gusto en las actividades y ser agradecido de tener las habilidades y el interés por forjar un camino dedicado al servicio de las personas".

\section{DISCUSIÓN}

Este estudio, construido desde la participación activa de un grupo de estudiantes que ingresan al primer año de Terapia Ocupacional, nos brinda la posibilidad de evaluar el desempeño ocupacional considerando la complejidad de factores que involucra el desarrollo del rol de estudiante en una facultad de altas exigencias académicas.

Cada vez resulta más evidente la necesidad de que los profesionales en formación desarrollen a lo largo de su carrera, no sólo competencias duras o cognitivas, sino además, habilidades sociales, ya que éstas influirán en gran medida en su desarrollo profesional y empleabilidad. (Alucio, G. Revellino 2011). Este trabajo permite a los jóvenes en formación tener una instancia académica de reflexibilidad sobre su situación en el mundo.

Investigaciones acerca del desempeño ocupacional de los estudiantes de terapia ocupacional (Blanco, G. $2012)^{1}$ nos indican que se puede establecer que existe influencia del estrés académico y de los componentes que lo conforman sobre el desempeño ocupacional del estudiante, ya que afectan de manera no satisfactoria la ejecución de las áreas de desempeño, roles y su participación activa en los diferentes contextos, quebrantando así el equilibrio vital, entendiéndose éste, como el equilibrio entre los factores biopsicosociales que toda persona desea alcanzar a lo largo de su vida.

Los resultados también permiten sugerir que se estudie la posibilidad de modificar el pensum de estudio en cuanto a la distribución del número de asignaturas por año y carga horaria, así como modificar el modelo educativo tradicional de horas presenciales en el aula, en el cual el estudiante además de cumplir con la carga horaria establecida por el pensum, debe realizar una serie de actividades fuera de las horas de clase, afectando de esta manera el tiempo que puede ser utilizado para otras actividades. 
Es interesante mencionar que las investigadoras presentaron en el IV Congreso Latinoamericano de Terapia Ocupacional, la investigación "Patrón de Idiosincrasia de los alumnos que ingresan a Terapia Ocupacional", realizado en el año 2000, donde los resultados fueron congruentes con los estudios sobre el tema en otros países (Cynkin, 1990). Aunque en esta investigación se observa el empleo de la misma cantidad de tiempo en dormir y en estudio, existe aumento considerable de horas en AVDB, relacionadas principalmente con alimentación, y disminución de horas en actividades de tiempo libre.

Lo anterior llama la atención por lo que sería importante investigar, relacionando esta situación con factores socioculturales y socioeconómicos.

\section{REFERENCIAS BIBLIOGRÁFICAS}

Alucio, G.; Revellino, M. Relación entre autoeficiencia, autoestima, Asertividad y rendimiento académico en estudiantes que ingresaron a Terapia Ocupacional el año 2010. Revista Chilena de Terapia Ocupacional. 2011/ 1: p. 11 - 22.

AOTA (2002). Occupational Therapy Practice Framework: domain and process. AJOT, 56: 609-639.

Baudouin Jean-Michel. Elementos de historia para el trabajo bibliográfico y la formación crítica: agentividad y reflexión. En: Aprendizaje permanente. Competencias para una formación crítica: aprender a lo largo de la vida. Manuel Martí Puig y Jesús Gil Gómez compiladores. Edicions del Crec, 2012 Valencia.

Bateson, M. C. (2001). Composing a life. Grove Press.

Blanco, G. Estrés y desempeño ocupacional en estudiantes de terapia ocupacional. Revista Chilena de Terapia Ocupacional. 2012/ 1: p. 11 - 21.

Cymkin S. Robinson AM. (1990). "Occupational Tehrapy and Activities Health: Howard Elath through activities". Little, Brown Company. Boston.

Giannini, H. La reflexión cotidiana. Hacia una arquelogía de la experiencia. Santiago, editorial Universitaria. 1987.

Gómez S., Rueda L., Muñoz C. Patrón de Idiosincrasia de los alumnos que ingresan a Terapia Ocupacional. Bases para el Desarrollo de la Ciencia de la Ocupación. Facultad de Medicina de la Universidad de Chile. 2002.

Gonçalves, H. Corpo doente: Um estudo sobre a percepção corporal da tuberculose. In: Doença, Sofrimento, Perturbação: Perspectivas Etnográficas (L. F. Duarte \& O. F. Leal, org.). Rio de Janeiro: Fiocruz, 1998: 105-120.

Heller, A. Sociología de la vida cotidiana. Barcelona: Ediciones Península. 1998.
Martins, J.; Bicudo, M. A. V. A pesquisa qualitativa em Psicologia. São Paulo: Moraes, 1989.

Maturana, H. R. (1994). La ciencia y la vida cotidiana: La ontología de las explicaciones científicas. En P. Watzlawick y P. Krieg, (comps), El ojo del Observador. Contribuciones al constructivismo. Barcelona: Gedisa.

Matuska, K. Validity Evidence for a Model and Measure of Life Balance. 2010. Disponible en: http://conservancy.umn.edu/bitstream/93965/1/Matuska umn 0130E 11207.pdf

Matuska, K.; Heinz, A.; Jonsson, H.; Pentland, W.; Hakansson, C.; Davis, J.; Backman, C. Life balance: Multidisciplinary theories and research. 2010. Presentado en el XV Congreso Internacional de la Federación Internacional de Terapia Ocupacional. WFOT. Stgo. Chile.

Matuska, K.; Christiansen, C.; Polatajko, H. \& Davis, J. (Eds.). Conciliación de la vida: Teorías e investigaciones multidisciplinarias. 2009. AOTA Press, Bethesda, MD EE.UU, ISBN 978-1-55642906-4, Capítulo 4, pp. 33-42.

Romero-Salazar, A. (1990). Diversidad o especificidad del análisis sociológico. Revista Contextos. 1990: 40-43.

Valdés, E. (2008). Humberto Giannini o la travesía desértica del ser ahí cotidiano. Revista Derecho Humanidades No 13. 141-156.

Villegas, M. González, F. (2011). La investigación cualitativa de la vida cotidiana. Medio para la construcción del conocimiento sobre lo social a partir de lo individual. Revista Psicoperspectivas. Individuo y Sociedad, Venezuela. Vol. 10, No. 2. 